\title{
Audit of guidelines for effective control of chemotherapy and radiotherapy induced emesis
}

\author{
Annabel B M Foot, Corinne Hayes
}

\begin{abstract}
A system was devised to establish the optimum treatment for emesis for each individual child receiving cytotoxic treatment. Cytotoxic drugs were ranked on a scale (1-5), with antiemetic regimens correspondingly graded. An age division $(\leqslant 5$ years, $>5$ years) was included. Cytotoxic treatment was given with coadministration of the parallel antiemetic regimen. Failure to control emesis required administration of a stronger regimen as defined in the guidelines.

A prospective clinical audit was performed to monitor the efficacy and utility of the system using diary cards to record episodes of nausea or vomiting, or both, completed by the patient or a parent and the nursing staff. The following audit criteria were set: (a) $80 \%$ control with first courses of chemotherapy; (b) $85 \%$ control with subsequent courses of similar chemotherapy; and (c) $90 \%$ lack of anticipatory nausea. Sixty children ( $<18$ years) received emetogenic cytotoxic drugs from February-June 1993. The criteria were satisfied in two of three categories, with $82 \%$ control for first courses of chemotherapy, $83 \%$ control for subsequent courses of chemotherapy, and $90 \%$ lack of anticipatory nausea. The guidelines were workable and acceptable overall. Minor modifications have been made subsequent to the audit to improve their efficacy further.

(Arch Dis Child 1994; 71: 475-480)
\end{abstract}

Chemotherapy induced emesis is a well recognised complication of the treatment of childhood malignancies. With the current trend towards more intensive treatment schedules it has the potential to impinge greatly on the quality of life of these children.

Recent advances in the field of $5-\mathrm{HT}_{3}$ receptor antagonists have assisted greatly towards improved antiemetic control. ${ }^{1-4}$ Such drugs will not be indicated in every case, however. Cytotoxic drugs vary considerably in their emetogenic potential, ranging from nonemetogenic compounds (for example, vincristine) to highly emetogenic treatment (for example, cisplatin). The influence of age must also be considered, with younger children observed to be more tolerant of emetogenic cytotoxic drugs than older children and adults. Finally, budget restrictions imposed on the individual institution may not allow the universal prescription of the relatively expensive $5-\mathrm{HT}_{3}$ receptor antagonists.

Most centres where children are treated for malignancies will have evolved in-house antiemetic protocols. Flexibility in prescribing to adjust to the individual patient's needs may not be standard practice, however. In addition, such changes are often the responsibility of relatively inexperienced staff. We therefore devised a graded system incorporating standardised antiemetic regimens currently used in our hospital, allowing modifications to suit the individual in a logical stepwise fashion. To assess both the serviceability and efficacy of these guidelines, a prospective criteria based audit was performed.

\section{Subjects and methods}

The system to be analysed took the form of a modified algorithm (fig 1). Cytotoxic drugs commonly encountered in the treatment of paediatric malignancies were categorised according to emetogenic potential from low (level 1) to high (level 5). Antiemetic regimens were correspondingly ranked from low (level 1 : no antiemetic treatment) to high (level 5: combination antiemetic treatment), with the addition of a 'rescue' combination (level 6) for treatment failure occurring at level 5 . Antiemetic regimens were stratified further according to age (A, >5 years; $\mathrm{B}, \leqslant 5$ years). The guidelines were applied by prescribing the antiemetic regimen corresponding to the level of the most emetogenic cytotoxic drug given each day. In the event of failure at that particular level, stronger antiemetic treatment was given by advancing to the next level.

All children less than 18 years of age attending Bristol Children's Hospital for the administration of cytotoxic treatment between 1 February 1993 and 30 June 1993 were eligible for assessment by audit. Monitoring of emetic control was performed with the aid of diary cards distributed to the nursing staff and the patient or a parent. These cards recorded the number of episodes of vomiting and retching (both diary cards) and nausea (patient card only) for each 24 hour period when chemotherapy was administered and for 48
Correspondence to:

Dr Foot.
Paediatric Oncology Royal Hospital for Sick Hill, Bristol BS2 8BJ A B M Foot 


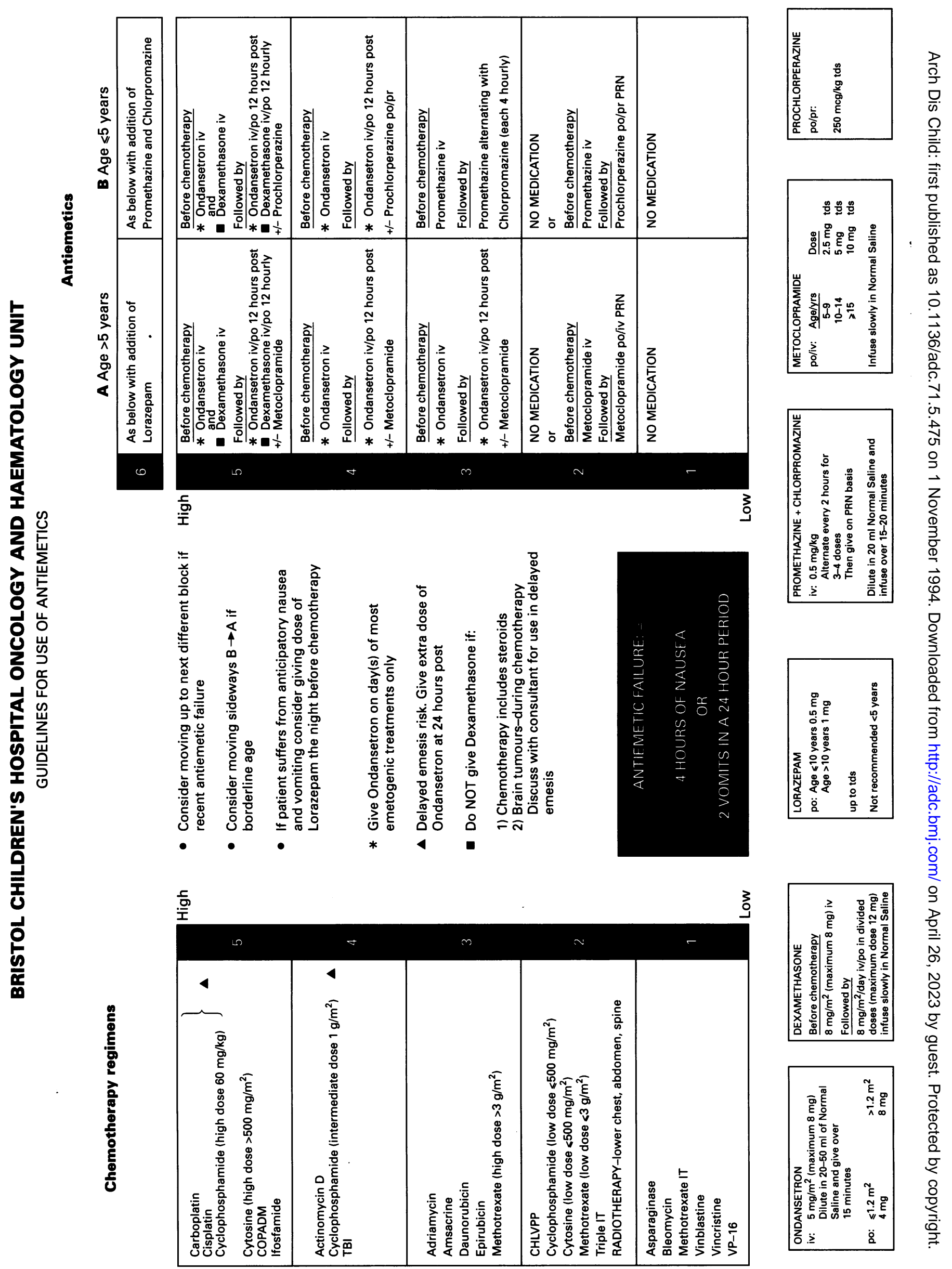

Figure 1 Guidelines for use of antiemetic drugs: audited version. TBI=total body irradiation. 
Table 1 Patient/parent held diary card

Date/day number

1 How many times did you/your child vomit (be sick) today?

2 How many times did you/your child retch (vomit without bringing anything up) today?

3 How severe has you/your child's nausea been today?

(1) Less than one hour

(2) One to four hours 4 Did you/your child suffer with symptoms of nausea shortly

5 Did you/your child suffer with symptoms of retching or vomiting shortly before treatment started?

Nursing held diary card as above (inserting patient for you/your child), but omitting questions 3-5 and including further information about cytotoxic and antiemetic treatment administered.

hours after the end of chemotherapy (table 1). For outpatient treatment, only the diary card held by the patient was completed.

Lack of control - that is, antiemetic failure was defined as two vomits/retches or four hours of nausea in a 24 hour period. One episode of vomiting or retching was defined as a single vomit, a single retch, or any number of continuous vomits, retches or heaves, each episode separated by no vomiting or retching for at least five minutes. For the purpose of the audit, antiemetic failure was recorded if either one or both diary cards documented lack of control.

\section{AUDIT CRITERIA}

The aim of this prospective audit was to assess the efficacy of the guidelines in terms of the control of emesis. It was defined that this would be achieved if the following criteria were met: (a) $80 \%$ control of emesis with first courses of chemotherapy; (b) $85 \%$ control with subsequent courses of similar chemotherapy; and (c) $90 \%$ lack of anticipatory nausea.

\section{Results}

Sixty children undergoing treatment for solid and haematological malignancies, including bone marrow transplantation, received emetogenic treatment over the time span of the audit. These children ( 35 boys, 25 girls) were aged $0.5-17.8$ years (median 6.5 years) and included 33 previously treated and 27 newly diagnosed children. In total, they received 164 courses of chemotherapy, $154(94 \%)$ of which were audited satisfactorily. Of these 154 courses, there was deviation from the guidelines in 12 courses and emetogenic factors other than cytotoxic treatment in a further eight courses. As data arising from these 20 courses would not assess the efficacy of the guidelines and emesis induced by cytotoxic drugs, it was excluded from the audit. The final analysis was therefore undertaken on the remaining 134 courses. The duration of the courses ranged from a single day to 10 days (median duration three days). This amounted to a total of 303 days of cytotoxic chemotherapy. Emetogenic treatment given to this cohort of children (a child often receiving more than one drug on single or repeated occasions over this time period) included cyclophosphamide (34 courses), cytosine (33 courses), methotrexate (27 courses), total body irradiation (13 courses), epirubicin (11 courses), actinomycin D (10 courses), daunorubicin (nine courses), Adriamycin (eight courses), cisplatin (eight courses), ifosfamide (six courses), carboplatin (six courses), and amsacrine (two courses).

\section{AUDIT}

Overall

Antiemetic control as previously defined was recorded in $110(82 \%)$ of the 134 courses. When analysed with respect to total chemotherapy days, control was achieved on $270 / 303(89 \%)$ days and included $218(72 \%)$ days which were entirely free of any episodes of nausea or vomiting (table 2 ).

\section{First course of chemotherapy}

Antiemetic regimens were prescribed for cytotoxic treatment for the first time according to the guidelines in $88(66 \%)$ of the total 134 courses. Antiemetic control was achieved for $72(82 \%)$ courses and 214/239 (90\%) days.

\section{Repeated courses of chemotherapy}

A second or further course of similar chemotherapy was administered on the remaining 46 occasions, with antiemetic control documented for $38(83 \%)$ courses and $56 / 64(88 \%)$ days. It should be noted, however, that four of eight course failures (corresponding to four of eight day failures) were recorded in the same patient. This was an adolescent with anticipatory symptoms receiving treatment for relapsed disease, but refusing appropriate drugs (see also later sections). If the data from this patient are removed from the analysis (on the grounds that it was not possible to adhere strictly to the suggested guidelines), the course success rate and day success rate become $90 \%$ and $93 \%$ respectively.

\section{Course failures}

Of the 16 children recording course failures with the first course of chemotherapy, five children received further similar treatment during the audit. Three of these children achieved control with the second course of treatment while applying guideline recommendations, and a fourth child required two adjustments before achieving control. The remaining child, with anticipatory symptoms and non-compliant as outlined in the previous section, was not treated successfully.

Table 2 Results of audit; values are number (\%)

\begin{tabular}{lcc}
\hline & Course control & Day control \\
\hline A & $110 / 134(82)$ & $270 / 303(89)$ \\
Overall & $72 / 88(82)$ & $214 / 239(89)$ \\
First course & $38 / 46(83)$ & $56 / 64(88)$ \\
Subsequent courses & $6 / 60(10)$ children \\
\hline Anticipatory nausea & \multicolumn{2}{l}{} \\
\hline
\end{tabular}




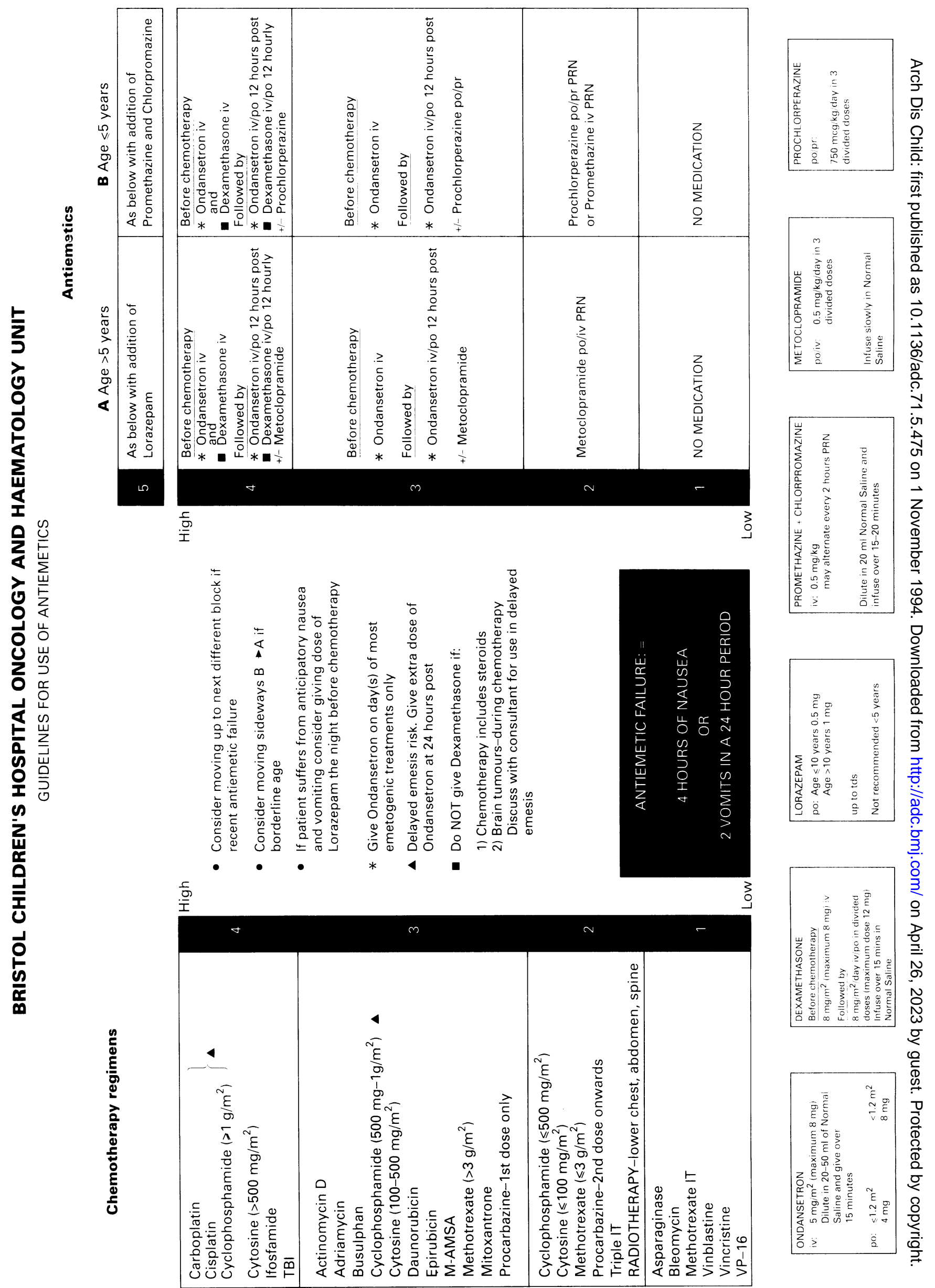




\section{Anticipatory nausea}

The patient/parent held diary cards included a brief section to document the presence of anticipatory symptoms before each course of chemotherapy. Six $(10 \%)$ children reported anticipatory problems, but these resolved in five patients with the appropriate alteration of antiemetic treatment as set out in the guidelines. The sixth patient (discussed earlier) refused medication.

\section{Delayed vomiting}

Delayed vomiting was assessed by recording episodes of vomiting/nausea for a further 48 hours after the end of chemotherapy. Lack of control was reported after treatment containing cyclophosphamide (one of 34 courses assessed in total), ifosfamide (one of six courses), methotrexate (two of 27 courses), and actinomycin (two of 10 courses), one course containing two of the listed drugs. Lack of emetic control had previously been reported in all five patients.

\section{ANALYSIS OF UNSUCCESSFUL CONTROL IN CHEMOTHERAPY}

Further scrutiny of the diary cards was performed in an effort to identify level allocation failure of specific cytotoxic treatment. The following were identified: level 2 , cytosine $300 \mathrm{mg} / \mathrm{m}^{2}$ (3/3 failures); and level 4 , total body irradiation (1440 cGy, eight fractions) (6/13 failures).

\section{ASSESSMENT OF PROTOCOL B ( $\leqslant 5$ YEARS)}

In view of the fact that infants were given specific and differing guidelines, this group, containing 24 children, underwent further analysis. In general, the problems encountered were similar to those already described. It was noted, however, that the option to transfer from level $3 \mathrm{~B}$ to level $3 \mathrm{~A}$ was commonly taken (17 of 22 occasions) and not restricted to those patients of borderline age only. This was recorded as staff preference of ease of administration (a 12 hourly rather than a two hourly regimen), particularly during prolonged infusions of cytotoxic treatment.

\section{Discussion}

The mechanism of the emetic response is complex, with several sites of action identified and differing antiemetic drugs indicated. ${ }^{5}$ Although there are many reports of the efficacy of various antiemetic regimens in adults, there is surprisingly little about administration specifically in children. In addition, a survey of paediatric antiemetic practice suggests that the choice of drugs is based on experience rather than clinical trials, and varies considerably from centre to centre. ${ }^{6}$ The widespread use of phenothiazines, in particular, is reported. ${ }^{6}$ Steroids have been shown to be effective in the control of moderate emesis, ${ }^{7}$ and may also be used in combination regimens for highly emetogenic drugs. ${ }^{3}$
Metoclopramide administration in children and young adults is unfortunately associated with a relatively high incidence of extrapyramidal reactions, particularly when used in high doses, which obviates its widespread use. ${ }^{89}$ The addition of $5-\mathrm{HT}_{3}$ receptor antagonists to the antiemetic repertoire has been encouraging, with much work reported on their use in adults and children. ${ }^{1-4}$ Dosage schedules vary, but studies in adults and preliminary studies in children suggest that twice daily administration of ondansetron is as effective as more frequent doses. ${ }^{10}$ In practice, however, it is not usually single drugs but combinations of treatment that provide the best antiemetic control in difficult cases.

The antiemetic schedules applied in these guidelines incorporated in-house regimens used before the introduction of the guidelines. This ensured familiarity of prescription and delivery at this institution, but may not be universally suitable in other centres. The basic format of the guidelines is such, however, that adaptation to incorporate local policies may be easily achieved.

The audit was undertaken for several reasons. Firstly, the efficacy of the guidelines required analysis. Although the audit criteria set were fairly severe, it is gratifying to note that they were fulfilled in two, and borderline in the third, with the overall levels of antiemetic control well within acceptable limits. The identification of level allocation failures has allowed modifications to be made in the final set of guidelines now in use at our institution (fig 2). Secondly, the serviceability and general acceptance by the medical and nursing staff required assessment. Although there were several errors made in the initial phase of the audit (these episodes therefore being excluded from the analysis as described), they decreased with time and experience. The main area of dispute was level $3 \mathrm{~B}$ for the infants, proving cumbersome and time consuming for nursing staff to administer. This level has therefore been omitted from the revised guidelines, and this simplification, together with several minor additional alterations, has resulted in the guidelines in current use (fig 2).

It is highly desirable to maximise antiemetic treatment in every patient. Often such treatment is prescribed in a non-standardised fashion by junior staff with little experience of oncology. Failure early in the course of a treatment schedule may then be followed by anticipatory symptoms, making for more difficult control. Standardised guidelines provide consistency and have been a great asset in many fields of medical practice. With increasing numbers of available antiemetic drugs and more emetogenic cytotoxic regimens, this was a field we saw to benefit from such a set of guidelines. Although we have incorporated antiemetic regimens previously used in our department, the guidelines could easily be adapted to suit each individual institution, provided they are then assessed adequately by audit. We are pleased to be able to report their efficacy in our practice. 
We thank the nursing and medical staff in the department of paediatric oncology, Royal Hospital for Sick Children, Bristol for their cooperation with this study. We thank the Glaxo oncology support team for their assistance with provision of oncology support tear

1 Pinkerton CR, Williams D, Wootton C, Meller ST, McElwain TJ. 5-HT3 antagonist ondansetron - an effective outpatient antiemetic in cancer treatment. Arch Dis Child 1990; 65: 822-5.

2 Hewitt M, Cornish J, Pamphilon D, Oakhill A. Effective emetic control during conditioning of children for bone marrow transplantation using ondansetron, a 5- $\mathrm{HT}_{3}$ antagonist. Bone Marrow Transplant 1991; 7; 431-3.

3 Smith DB, Newlands ES, Rustin GJ, et al. Comparison of ondansetron and ondansetron plus dexamethasone as antiemetic prophylaxis during cisplatin-containing chemotherapy, Lancet 1991; 338: 487-90

4 Stevens RF. The role of ondansetron in paediatric patients: a review of three studies. Eur $\mathcal{F}$ Cancer 1991; suppl 1: S20-2.

5 Grunberg SM, Hesketh PJ. Control of chemotherapyinduced emesis. $N$ Engl $\mathcal{F}$ Med 1993; 329: 1790-6.

6 Van Hoff J, Hockenberry-Eaton MJ, Patterson K, Hutter JJ. A survey of antiemetic use in children with cancer. $A m \mathcal{F}$

7 Mehta P, Gross S, Graham-Pole J, Gardner R. Methylprednisolone for chemotherapy-induced emesis: a double-blind randomised trial in children. $\mathcal{F}$ Pediatr 1986; 108: 774-6.

8 Terrin BN, McWilliams NB, Maurer HM. Side-effects of metoclopramide as an anti-emetric in childhood cancer chemotherapy. $\mathcal{f}$ Pediatr 1984; 104: 138-40.

9 Allen JC, Gralla R, Reilly L, et al. Metoclopramide: dose related toxicity and preliminary anti-emetic studies in children receiving cancer chemotherapy. 7 Clin Oncol 1985; 3: 1136-46.

10 Proceedings of the ondansetron satellite symposium to the 15th International Cancer Congress (IUCC), Hamburg, August 1990. Eur 7 Cancer 1991; 27 (suppl 1). 